Meta

Journal des tradlucteurs

Translators' Journal

\title{
Apport au problème de la synonymie
}

\section{Hans-Martin Gauger}

Volume 15, numéro 3, septembre 1970

URI : https://id.erudit.org/iderudit/002823ar

DOI : https://doi.org/10.7202/002823ar

Aller au sommaire du numéro

Éditeur(s)

Les Presses de l'Université de Montréal

\section{ISSN}

0026-0452 (imprimé)

1492-1421 (numérique)

Découvrir la revue

Citer cet article

Gauger, H.-M. (1970). Apport au problème de la synonymie. Meta, 15(3),

147-160. https://doi.org/10.7202/002823ar d'utilisation que vous pouvez consulter en ligne.

https://apropos.erudit.org/fr/usagers/politique-dutilisation/ 


\section{Apport \\ au problème \\ de}

la synonymie

Dans un mot, on doit distinguer deux choses : une forme phonique, composée d'un ou de plusieurs «phonèmes», et une autre chose, non phonique et moins facile à saisir, que nous appelons le «contenu». Le contenu est «lié » à la forme phonique dans la conscience des sujets parlants. C'est par cela - et par cela seulement - que la succession de sons devient un MOT, c'est-à-dire un sIGNe qui figure comme NoM pour quelque chose d'autre que lui-même : aliquid stat pro aliquo, comme disaient les scolastiques.

C'est donc grâce à son contenu que le mot figure comme nom pour une chose déterminée, que celle-ci soit réelle ou imaginée. Plus exactement, en termes empruntés à la logique : il sert de nom pour une «classe » de choses. Car la langue ne désigne pas, en général, des objets particuliers. Elle rassemble ce qu'elle reconnaît dans le monde comme des "choses 》 distinctes, selon des critères qui ne sont pas toujours facilement discernables, créant ainsi des «classes ». L'objet particulier - un arbre particulier, une maison particulière - apparaît ainsi comme membre d'une «classe». Ceci vaut pour ce qu'on appelle les «noms communs 》 (nomina appellativa). La logique plus récente parle ici de «prédicateurs » et entend par ce terme tout ce qui peut être attribué à un « objet », sans se soucier si, du point de vue de la langue, il s'agit d'un substantif, d'un verbe ou d'un adjectif : « $x$ est un arbre », " $x$ dort », « $x$ est rouge ». Nous ne parlerons ici que de noms communs. Pour saisir le sens de ces mots, il n'est d'ailleurs pas indispensable d'avoir recours au concept de «classe » tel qu'il a été développé par la logique mathématique. On peut même se demander si on a le droit d'y faire appel. En général, l'introduction d'éléments de la logique dans la recherche linguistique ne montre ce qu'est la langue que dans la mesure où elle montre ce qu'elle n'est pas. Si l'on rappelle très souvent les vers du Faust :

Mein teurer Freund, ich rat euch drum, zuerst Collegium Logicum ${ }^{1}$,

1. "Mon cher ami, c'est pourquoi je vous conseille : d'abord le cours de logique. » 
on ne devrait pas oublier que c'est Méphistophélès qui donne ce conseil : comme tous les conseils venant de ce côté-là, celui-ci est intéressant, mais doit être accueilli avec réserve. En ce qui concerne notre problème, le linguiste peut se contenter de dire que, par des mots comme maison et arbre la langue entend « des choses comme... » ( une maison est une chose comme celle que tu vois ici »), et que ce «comme » est saisi d'une manière intuitive par le sujet parlant dans l'emploi concret de ces mots, dans la praxis du langage. Ce procédé abstractif dans la désignation des objets apporte à la langue un inimaginable bénéfice économique. D'autre part, ce n'est que grâce à lui que la phrase réussit à devenir ce qu'elle est pour l'homme : un instrument à la fois d'expérience du monde et de prise de possession du monde.

On sait que chaque « chose » vue par la langue ne reçoit pas comme nom un seul signe phonique particulier, et rien que celui-là. La situation se complique dans la mesure où un même signe peut désigner différentes choses et où des signes différents peuvent désigner la même chose. On parle généralement de POLYSÉMIE, quand un seul mot est rattaché à deux ou plusieurs contenus, c'est-à-dire quand un seul mot désigne deux ou plusieurs choses différentes. On parle de sYNONYMIE, quand deux ou plusieurs mots sont rattachés à un seul contenu, donc quand deux ou plusieurs mots désignent une seule et même chose. Voilà, dans leur essence, les renseignements fournis par les manuels, par les excellents manuels de Stephen Ullmann par exemple ${ }^{2}$. Polysémie : un seul mot, deux ou plusieurs contenus. Synonymie: deux ou plusieurs mots, un seul contenu. Les deux phénomènes c'est là le point décisif - sont considérés comme étant op POSÉs l'un à l'autre.

Nous porterons, dans cet article, notre attention sur la synonymie, sans nous occuper de la polysémie ni de l'homonymie (cette dernière est considérée généralement comme la forme la plus radicale de la polysémie ${ }^{3}$ ). Voici la question que nous posons au départ : est-il justifié d'entendre par «synonymes» des mots qui ont le même contenu ? Dans ce cas, ces mots ne devraient-ils pas être interchangeables, chaque fois que l'un d'entre eux apparaît quelque part, et cela, sans la moindre modification dans le contenu global de l'énoncé ? C'est précisément ce que dit la définition d'Ullmann : «Only those words can be described as synonymous which can replace each other in any given context, without the slightest alteration either in cognitive or in emotive import ${ }^{4} . »$ On rencontre une définition très semblable dans le livre de John Lyons : «Two (or more) items are synonymous if the sentences which result from the substitution of one for the other have the same meaning ${ }^{5}$. $\gg$ Qu'est-ce qui n'est pas satisfaisant dans ces définitions?

Ces définitions sont « vides » : en fait il n'existe pas de «synonymes » correspondant à ces définitions. En comparant les contenus des mots dits «synonymes », on découvre toujours entre eux des différences plus ou moins marquées, quoique souvent difficilement exprimables. Jusqu'ici, personne (les auteurs cités pas plus

2. The Principles of Semantics, Oxford, 1951; Précis de sémantique française, Berne, 1952; Semantics. An Introduction to the Science of Meaning, Oxford, 1964.

3. Cf. S. Ullmann, Principles, p. $177 \mathrm{~s} ;$ p. $125 \mathrm{~s} ; \mathrm{K}$. Heger propose une solution nouvelle : Homographie, Homonymie und Polysemie »; Zeitschrift für romanische Philologie, 79 (1963), p. 471-491.

4. S. Ullmann, Principles, p. 108-109.

5. Introduction to Theoretical Linguistics, Cambridge, 1968, p. 428. Pour Lyons la synonymie est un cas spécial de ce qu'il appelle l' "hyponymie " (p. 455). 
que les autres) n'a fourni un exemple convaincant de parfaite identité de contenu. Il semble n'exister de synonymes parfaits que dans le domaine des langages techniques; dans les langues naturelles, les langues qui se sont formées historiquement, on n'en trouve pas. D'autre part, c'est traditionnellement la «synonymique » qui s'occupe de ce qu'on appelle les synonymes; elle cherche à dégager les différences existant entre les contenus des synonymes; elle cherche la différence dans ce qui est semblable. Son objet est donc précisément la différence des contenus et non pas leur identité. La pure identité ne demanderait pas de recherches du genre de celles pratiquées dans la «synonymique »: à son sujet, il n'y aurait rien à remarquer. $\mathrm{La}$ « synonymique », donc, parle de quelque chose que la définition du terme «synonyme » veut précisément exclure.

Cette «synonymique » est née en France au début du XvIII ${ }^{e}$ siècle. Dès ses débuts, elle prit comme point de départ la conviction qu'il n'existait pas de mots ayant à tous points de vue le même sens. Son fondateur est le grammairien Gabriel Girard (1677-1748). Et c'est déjà lui qui déclare dans le « Discours préliminaire » de son livre : « je ne crois pas qu'il y ait de mot synonyme dans aucune langue... je suis très persuadé et j'ose presque dire assuré qu'il n'y en a point dans la nôtre ${ }^{6}$. $\gg$ C'était même le but déclaré de l'abbé Girard de démontrer qu'il n'existait pas de synonymes véritables, du moins dans une langue évoluée comme la langue française. Sa synonymique, parue en 1718 , devint un livre particulièrement répandu, beaucoup lu et fort élogieusement commenté (Voltaire : "Son livre de Synonymes est très utile; il subsistera autant que la langue et servira même à la faire subsister ${ }^{7} \gg$ ). Le livre porte ce titre caractéristique : la Justesse de la langue françoise, ou les différentes significations des mots qui passent pour synonymes. Le terme « justesse » signifie ici que chaque mot de la langue française contient une signification très précise qui n'appartient qu'à lui seul et ne peut être rendue par aucun autre mot. Quand les mots sont différents, les significations le sont aussi : c'est cela qui fait la « justesse » du français, et constitue sa vraie richesse. Cette opinion fut aussi celle des nombreux successeurs de Girard au cours du XVIIr ${ }^{\mathrm{e}}$ et du XIX ${ }^{\mathrm{e}}$ siècle. Il s'agit donc d'une conception nouvelle de la « richesse » d'une langue : elle ne réside pas dans la copia verborum, mais dans la précision et la finesse des distinctions synonymiques ${ }^{8}$.

Dire qu'il n'y a « au fond » pas de synonymes n'est donc pas du tout original. La synonymique traditionnelle est dominée par cette idée. Ce qui est curieux, c'est que la définition traditionnelle - synonymie comme identité de contenu - s'est affirmée à côté de cette constatation généralement admise sans presque rencontrer de résistance. Cette définition a largement contribué au fait que le phénomène linguistique fondamental de la similitude de signification et les intéressants pro-

6. Dans la préface de la troisième édition (1740), Girard s'exprime d'une manière encore plus décidée : «...il n'y a point de mots assez parfaitement synonymes pour avoir, dans toutes sortes d'occasions, une force de signification semblable. *

7. Euvres de Voltaire, éd. Beuchot, XIX, p. 118.

8. A partir de la deuxième édition (1736), le livre de Girard porte le titre suivant: Synonymes françois, leurs significations et le choix qu'il en faut faire pour parler avec justesse. Nous avons présenté la synonymique de Girard et celle d'un de ses successeurs les plus intéressants, Pierre Joseph André Roubaud (1730-1792), dans notre thèse (Die Anfänge der französischen Synonymik und das Problem der Synonymie. Tübingen, 1960) qui sera publiée en 1971. 
blèmes systématiques et stylistiques qui s'y rattachent n'ont pas retenu l'attention autant qu'ils l'auraient mérité 9 .

Occasionnellement, on s'est demandé pourquoi il n'existe pas de synonymes stricto sensu. Michel Bréal l'a déjà fait au cours du siècle dernier. C'est, selon lui, la « loi de la répartition», qui s'oppose à la synonymie parfaite : involontairement, les sujets parlants se basent sur la conception que des mots différents signifient des choses différentes. Par l'action de ce « sentiment » intuitif, on obtient tout naturellement une distribution chaque fois différente de «traits » sémantiques sur les différents synonymes. Il y a effectivement quelque chose de vrai dans cette affirmation ${ }^{10}$. Le fait qu'il n'y a pas de synonymes provient de la conception intuitive du MOT propre au sujet parlant. Comme, pour celui-ci, le mot a pour tâche de désigner une « chose », en la distinguant d'autres «choses », DIFFÉRENTS mots doivent désigner des « choses » DIFFÉrentes. Quoi qu'il en soit, nous pouvons nous baser sur le fait empiriquement constatable qu'il n'y a pas de synonymes dans le sens précisé auparavant.

Vu cet état de faits, il est préférable d'entendre, dès l'abord, par le terme de «synonyme », des mots ayant un contenu semblable. Il est clair que de pareils mots existent. La similitude des contenus peut signifier que ces mots - par exemple, beau et joli - veulent exprimer quelque chose de semblable, ou bien alors que ces mots veulent exprimer la même chose sous des aspects chaque fois différents, comme les mots mourir, décéder, s'éteindre rendent sous différents aspects le MÊME événement ou du moins un processus qui donne le même résultat. Par le terme «synonymité » nous entendons la relation de similitude, les rapports sémantiques qui existent entre de tels mots, et qui constituent, précisément, l'objet de la « synonymique $»$.

Le phénomène de la synonymie n'est compréhensible que si l'on distingue les synonymes apparaissant dans des énoncés concrets (Sprachäusserungen), et les synonymes considérés comme des éléments non actualisés de la «langue » (Sprachbesitz). En d'autres termes : il faut mettre à la base une distinction analogue à celle de Saussure entre «parole» et «langue» ou à celle de Chomsky entre «performance » et «compétence».

La «langue » (《compétence 》), objet véritable de la linguistique, constitue la somme de moyens linguistiques (c'est-à-dire les mots et ce qu'on appelle les «règles grammaticales ») qui sont «empreints», d'une manière ou d'une autre, dans la conscience du sujet parlant, dans cette ingens aula memoriae, comme dit Augustin. Elle doit être comprise comme un «savoir », comme la domination d'une certaine « technique » - historiquement formée — de la parole. C'est précisément dans ce sens-là que les Latins disaient : latine loqui, graece loqui, etc. : parler à la façon latine, selon la manière, la «technique » du latin.

On doit distinguer de la «langue », qui est un fait «supra-individuel », mieux encore : un fait «interindividuel», l'énoncé particulier et individuel, qui est un

9. Citons quelques travaux récents sur le problème, dans lesquels on trouvera d'autres références:- O. Duchaček, "Différents types de synonymes", Orbis, 13 (1964), p. 35-49; W. Müller, "Probleme und Aufgaben deutscher Synonymik", Die wissenschaftliche Redaktion, I (1965), p. 90-101; L. Söll, "Synonyme und Bedeutungsgleichheit " GermanischRomanische Monatsschrift (1966), p. 90-99.

10. Cf. M. Bréal, Essai de sémantique, 3e éd., Paris, 1904, p. 26-38. 
acte de «parole» ou de "performance». La grande difficulté qui se pose au linguiste est due au fait que la langue, vers laquelle tendent ses recherches et qui est la condition nécessaire (mais non suffisante) de chaque énoncé concret, n'est pas immédiatement accessible. Elle ne peut être dégagée qu'INDIRECTEMENT, grâce à un nombre aussi élevé que possible d'énoncés particuliers qui, en eux-mêmes, n'intéressent pas le linguiste en tant que tel (ils ne sont pour lui que du «matériel», rien qu'un moyen pour atteindre la langue).

Considérons d'abord, en partant de cette distinction, les synonymes dans la parole. Quand deux ou plusieurs synonymes apparaissent dans un énoncé, ils apparaissent nécessairement à l'intérieur d'un contexte et d'une situation. Le contexte, la situation, fixent la signification du mot, qui, en elle-même, est flottante : ce n'est que du contexte et de la situation qu'on peut la déduire avec quelque certitude. Car le contenu total d'un énoncé ou d'une phrase n'a pas été composé progressivement d'éléments de contenu isolés et déjà achevés, selon la conception naïve, pourtant très répandue, qui voit dans une langue quelque chose comme un jeu de cubes. $\mathrm{Au}$ contraire, il faut partir de l'idée que le sens total, ce qui veut être dit, est déjà présent AVANT ses éléments, les différents mots ou «morphèmes » (《 monèmes »). Surtout, la situation dans laquelle on parle, est déjà existante. Le choix de chaque élément composant est déjà guidé par le sens total qui l'a précédé. Ainsi, ces éléments reçoivent une fonction sémantique plus ou moins précise, qui leur est déléguée, dans le sens total d'un énoncé et dans sa situation. Ceci est valable pour le sujet parlant. Pour l'auditeur, ce n'est pas, tout simplement, le contraire qui se réalise. On ne doit pas s'imaginer la compréhension d'un énoncé comme si l'auditeur combinait successivement les «bouchées » sémantiques fournies par la personne qui parle, d'une quelconque façon, par exemple selon la « hiérarchie » des « constituants immédiats », jusqu'à ce qu'il ait finalement devant lui le contenu achevé de l'énoncé entier. Car l'auditeur, lui aussi, est déjà, en général, introduit partiellement dans ce sens total. Par ces réalités complexes, que nous ne devons pas décrire plus en détail ici, une théorie de la langue qui s'oriente constamment et exclusivement selon le principe compositionnel, selon le principe du jeu de cubes, est condamnée inéluctablement à l'échec.

En ce qui concerne les synonymes dans la parole on doit distinguer entre deux sortes de contextes tout à fait différents. Dans un cas nous parlons de contextes SYNONYMIQUES, dans l'autre de contextes NON SYNONYMIQUES.

Le contexte non synonymique est le contexte ordinaire dans la parole. Les synonymes n'y apparaissent pas comme des synonymes selon notre définition, c'est-àdire qu'ils ne sont pas "thématisés » selon leur ressemblance et leur diversité. Ils apparaissent ici de telle sorte que ni le sujet parlant, ni l'auditeur ne prennent conscience des différences sémantiques qu'ils comportent. Il faut bien remarquer que dans la conversation normale et, en particulier, quand on parle avec concentration, ni la forme des mots, ni leur contenu ne sont ressentis consciemment en tant que tels. Du signe acoustique, l'attention saute aussitôt vers la chose désignée. Cet état de choses, qu'on pourrait qualifier d' « évacuation du contenu» (Inhaltsentleerung), a déjà été caractérisé par Leibniz dans son essai intitulé : "Unvorgreifliche Gedanken betreffend die Ausübung und die Verbesserung der deutschen Sprache. » Selon Leibniz on utilise les mots comme des « jetons de l'esprit» (Wechsel-Zeddel des 
Verstandes). D'après lui on ne se fait pas, chaque fois qu'on les prononce ou qu'on les entend, une «véritable image » de leur sens ${ }^{11}$. Un important concept de la psychologie de Sigmund Freud peut être introduit ici, celui du «préconscient» (Vorbewusstes). Ce dernier concerne cet important domaine du psychisme entre ce qui est effectivement conscient (Bewusstes) et ce qui est vraiment et définitivement inconscient (Unbewusstes). Les mots, et donc aussi les synonymes, restent et agissent dans les contextes «non synonymiques», dans le domaine du préconscient; c'est-à-dire qu'ils sont bien, en principe, en état d'être réalisés consciemment, ils pourraient devenir conscients, mais ils restent, en général, effectivement inconscients ${ }^{12}$

Il en va tout autrement dans les contextes «synonymiques » : ici, les synonymes sont «thématisés » justement comme des mots au sens semblable, c'est-à-dire aussi comme des mots de sens plus ou moins différent. Ici, on traite les synonymes comme des synonymes, donc au point de vue de leur sYNONYMiTé. Les contextes « synonymiques» impliquent un commentaire en quelque sorte métalinguistique : « avec le mot $\mathbf{A}$ je veux dire autre chose qu'avec le mot $\mathbf{B} \gg$. On pourrait appeler ce commentaire $\mathrm{l}$ ' «implicat de diversité ». Comme exemple je cite un aphorisme du Buch der Freunde de Hofmannsthal. Le voici : "Man schätzt diejenigen als etwas Seltenes, die ruhig und aufmerksam zuzuhören verstehen; ebenso selten ist ein wirklicher Leser, am seltesten aber ist einer, der seine Nebenmenschen auf sich wirken lässt, ohne den Eindruck unablässig durch seine innere Unruhe, Eitelkeit, Selbstsucht $z u$ zerstören, $j a z u$ vernichten ${ }^{13}$. »Ce contexte «synonymique 》 implique que vernichten ne signifie pas la même chose que zerstören, mais quelque chose de plus fort. L'indication de la diversité n'est que sous-entendue implicitement. Elle peut évidemment aussi apparaître explicitement. Sigmund Freud écrit dans une lettre concernant la question « Pourquoi la guerre ? » à Albert Einstein : «Sie beginnen mit dem Verhältnis von Recht und Macht. Das ist gewiss der richtige Ausgangspunkt für unsere Untersuchung. Darf ich das Wort « Macht» durch das grellere, härtere Wort "Gewalt » ersetzen ${ }^{14}$ ? \& Ici, la situation est plus compliquée, car la différence ne porte pas tellement sur les représentations des choses que sur les représentations des mots : la langue lie une représentation négative au mot Gewalt. Par ce fait, il signifie quand même autre chose que le mot Macht. Ce que la langue distingue de la chose Gewalt par le mot Macht, n'est finalement pas différent, selon Freud, de ce qu'elle appelle Gewalt; Macht n'est qu'un mot de camouflage pour Gewalt. Selon Freud, la langue allemande fait ici une distinction injustifiée.

Par le contexte « synonymique », les synonymes qui y apparaissent se trouvent

11. Cf. J. Stenzel, Philosophie der Sprache, Separatum, Handbuch der Philosophie, Munich, 1934 , p. 61; et H.-M. Gauger, Wort und Sprache, Sprachwissenschaftliche Grundfragen, Tübingen, 1970 , p. 76-79.

12. Sur le concept freudien du " préconscient" (Vorbewusstes), cf. S. Freud, Das Unbewusste, t. X des Gesammelte Werke; et H.-M. Gauger, op. cit., p. 32-37.

13. H. von Hofmannsthal, Buch der Freunde, Francfort, Insel-Bücherei, 1965, p. 7 : " On estime comme une rareté ceux qui savent écouter tranquillement et avec attention; il est tout aussi rare de trouver un véritable lecteur ; mais ce qu'on découvre le plus rarement, c'est quelqu'un qui laisse agir ses semblables sur lui, sans constamment détruire, voire anéantir, l'impression qu'ils font sur lui, par son agitation intérieure, sa vanité, son égoïsme *.

14. Warum Krieg?, t. XVI des Gesammelte Werke : "Vous commencez avec les rapports entre le droit et le pouvoir. Voilà certainement un point de départ correct pour nos réflexions. Puis-je remplacer le mot pouvoir par le mot plus cru, plus dur de violence? » 
actualisés d'une manière particulière : leurs différences, qui demeuraient voilées dans le contexte «non synonymique », apparaissent d'une façon saisissante dans le contexte "synonymique ». Comme tout le poids du sens de la phrase pèse sur eux, les synonymes se trouvent aussitôt dans le plein éclairage de la conscience. Le contexte «synonymique» amène ainsi une réalisation totale du sens, et celle-ci amène une mobilisation des différences incluses dans les synonymes. Le contexte "synonymique » est un renvoi conscient du sujet parlant (ou de celui qui écrit) vers la langue dont il dispose, et il cherche à provoquer chez l'auditeur (ou chez le lecteur) le même mouvement de réflexion.

On s'aperçoit ici qu'une langue humaine ne peut être pensée sans un sujet dont la conscience se fixe éventuellement sur elle. Il n'y a pas de langue sans conscience : la réflexivité, l'unité de langue «objective » et de «métalangue », est le trait constitutif de la langue «naturelle ${ }^{15}$ ».

Le contexte «synonymique » fait donc saillir les différences entre les synonymes, tandis que le contexte «non synonymique» fait précisément disparaitre ces différences. Il faut ajouter que ces contextes sont des types idéaux et ne doivent pas être réalisés nettement, dans un sens ou dans l'autre, dans chaque cas particulier. Le fait que, dans la parole, la langue peut «manipuler» les synonymes qui lui appartiennent de telle ou telle façon, n'a guère retenu l'attention, semble-t-il. C'est pourtant une des plus remarquables réalisations de la langue. Comme exemple d'une pareille manipulation nous citons, pour les deux synonymes éducation et instruction, un contexte «synonymique » et un contexte "non synonymique ».

Dans les Considérations sur les mours de ce siècle de Charles Duclos (17041772) nous lisons : «On trouve parmi nous beaucoup d'instruction et peu d'éducation ». Ce que nous avons appelé l' «implicat de diversité » est facile à saisir dans cette courte phrase : «éducation ne signifie pas la même chose qu'instruction». Si le lecteur veut percevoir le sens de la phrase, il doit se représenter consciemment les deux contenus et les confronter. Il se fait d'eux, comme disait Leibniz, une "véritable image ». Ceci l'amène à constater qu'éducation signifie la formation entière d'un homme, y compris la formation morale, tandis qu'instruction s'applique plutôt à la simple transmission de contenus plus ou moins importants. Cette différence incontestable ne ressort pas dans le contexte «non synonymique ». Fénelon écrit au sujet de l'éducation des jeunes filles : «Rien n'est plus négligé que l'éducation des filles. La coutume et le caprice des mères y décident souvent de tout ; on suppose qu'on doit donner à ce sexe peu d'instruction. L'éducation des garçons passe pour une des principales affaires... ${ }^{16} \gg$. Dans ce contexte « non synonymique » nous trouvons deux fois éducation et une fois instruction : ici nous ne nous sentons pas porté à noter des différences entre les contenus, et nous agissons ainsi selon les intentions de l'auteur, qui veut seulement, dans ces lignes, affirmer le fait que seuls les garçons bénéficient d'activités pédagogiques, tandis que les filles sont négligées.

15. Sur le rôle de la conscience dans la langue et dans la linguistique, cf. le premier chapitre : "La conscience linguistique et la linguistique", de mon livre Wort und Sprache, p. 1-44.

16. Duclos, Considérations sur les meurs de ce siècle, Paris, 1751, chap. II, p. 33. Le passage de Fénelon est cité dans le Dictionnaire de P. Robert. Cf. Stendhal, la Chartreuse de Parme, I, 2: "Le marquis professait une haine vigoureuse pour les lumières : ce sont les idées, disait-il, qui ont perdu l'Italie ; il ne savait trop comment concilier cette sainte horreur de l'instruction, avec le désir de voir son fils Fabrice perfectionner l'éducation si brillamment commencée chez les jésuites. 》 
Le poids du sens dans cet énoncé ne repose pas sur ces synonymes. En fait, Fénelon veut dire chaque fois exactement la même chose, ou plutôt : les sens sont ici "vidés», selon la conception de Leibniz. On fausserait en effet l'intention de l'auteur en évoquant consciemment les deux contenus en eux-mêmes et ainsi, nécessairement, leur diversité. Si l'on lit la phrase comme elle doit être lue, les différences entre éducation et instruction, qui s'imposaient spontanément dans la phrase de Duclos, doivent rester totalement dissimulées ici.

Nous n'indiquons qu'en passant que l' «évacuation du sens», qui ne peut en aucun cas être jugée négativement, est également importante pour la critique littéraire : celle-ci ne peut pas partir, comme elle le fait en général, de la conception que, dans un texte, chaque mot particulier, comme "gonflé à bloc», à été placé par l'écrivain, par le poète, avec tout le poids de son contenu et toutes les implications de celui-ci.

Citons, sans les commenter, quelques exemples, pris au hasard de la lecture, pour illustrer notre distinction entre les deux types de contextes. Il est évident qu'une étude systématique de tels contextes mènerait à une certaine différenciation de ce que nous n'avons pu qu'esquisser ici.

\section{Contextes synonymiques}

* La plupart des agonies sont euphoriques. Autre chose est de jeter bas d'un seul coup, d'une seule parole, tout l'espoir d'un homme... vos théologiens ont fait de l'espérance une vertu, votre espérance a les mains jointes. Passe pour l'espérance, personne n'a jamais vu cette divinité-là de très près. Mais l'espoir est une bête, je vous dis, une bête dans l'homme, une puissante bête, et féroce.» (G. Bernanos, Journal d'un curé de campagne, Paris, 1955, p. 230-231).

" Je ne sais ce qu'il dit : j'étais trop soucieux d'écouter pour entendre." (J.-P. Sartre, les Mots, Paris, 1964, p. 16).

«... la voix qui tremblait d'amour en m'appelant «cadeau du ciel», je feignais encore de l'écouter mais j'avais fini par ne plus l'entendre. Pourquoi lui ai-je prêté l'oreille ce jour-là, au moment qu'elle mentait le plus délibérément? (J.-P. Sartre, les Mots, p. 130).

"Longtemps j'ai envié les concierges de la rue Lacépède, quand le soir et l'été les font sortir sur le trottoir, à califourchon sur leurs chaises : les yeux innocents voyaient sans avoir mission de regarder. \$(J.-P. Sartre, les Mots, p. 136).

- La Mort brillait par son absence : décéder, ce n'était pas mourir, la métamorphose de cette vieillarde en dalle funéraire ne me déplaisait pas... » (J.-P. Sartre, les Mots, p. 77). "Le Rouge [il s'agit d'un torero], avec fureur, esquissa plutôt qu'il ne dessina quelques passes confuses... " (H. de Montherlant, le Chaos et la nuit, Paris, 1963, p. 254).

"... ce n'est pas de l'orgueil, c'est de la fierté légitime et très humble d'un saint... * (Bible de Jérusalem, Introduction aux épîtres de saint Paul, p. 1481).

"Pourquoi toujours revenir à ce mot ennemi! Faut-il vous le redire ? $\mathrm{Ce}$ ne sont pas les ennemis naturels qui se battent. Il est des peuples que tout désigne pour une guerre, leur peau, leur langue et leur odeur, ils se jalousent, ils se haïssent, ils ne peuvent pas se sentir... Ceux-là ne se battent jamais. Ceux qui se battent, ce sont ceux que le sort a lustrés et préparés pour une même guerre : ce sont les adversaires.» (J. Giraudoux, la Guerre de Troie n'aura pas lieu, Paris, 1935, p. 185).

"Le Français considère l'Allemand comme un être à part. Qu'il l'aime ou ne l'aime pas, peu importe, l'Allemand lui paraît étranger, voire étrange, beaucoup plus que les autres Latins, cela va sans dire, mais sans doute aussi beaucoup plus que les Anglais, les Américains et peut-être les Russes. " (L. Remy, Voici l'Allemagne nouvelle, Düsseldorf, 1967, p. 12). 
* Les ministres observent. En réalité peut-être conviendrait-il d'écrire: les ministres épient. 》 (Paris-Match, sur les réunions du conseil avec de Gaulle).

« Der eigenen Träne muss ich mich schämen, denn ich vergoss sie nur aus sanfter Wehmut, ihrer aber war ein Schmerz, den keine Träne lindert, und als sie zum zweitenmal erwachte, war ihr Auge trocken und ihre Miene starr.» (Th. Mann, Der Erwählte, Francfort, 1951, p. 74).

" Aber wie übersüss war's doch, als er bei mir war, als ich seine holde Schulter an meinen Lippen hatte und er mich, wenn nicht zur Frall, so doch zum Weibe machte! \$ (Th. Mann, Der Erwählte, p. 62).

« In dem Vortrag über Richard Wagner, mit dem ich, vor nun bald fünf Jahren, im Auditorium maximum der Münchner Universität, ohne es zu wissen oder zu ahnen, von Deutschland Abschied nahm... 》 (Th. Mann, * Richard Wagner und Der Ring des Nibelungen ", dans: Wagner und unsere Zeit, Francfort, 1963, p. 127).

"Sie war wirklich nur noch ein Bündel von Knochen und Haut, und ihr Kopf wurde zum Schädel. \(J. Maass, Der Fall Gouffé, Francfort, 1955, p. 61).

« Meine Schwester war ziemlich hübsch, manche Leute fanden sie schön... » (J. Maass, Der Fall Gouffé, p. 59).

« Ich verstehe es ausgezeichnet, meinen Kopf, so, als wenn ich über etwas empört, nein, nur erstaunt wäre, zurückzuwerfen. " (R. Walser, Jakob von Gunten, Munich, 1964, p. 56-57).

«Das lyrische Bewusstsein fängt sich im Netz, im Gitter, im Käfig der Worte, der Wörter. " (H. Weinrich sur le poète P. Celan, dans : Neue Rundschau, 1968, p. 115).

* La famiglia si affollava sulla porta : La Principessa aveva ritirato le proprie riserve dinanzi all'ira maritale, che le aveva, non è sufficiente dire respinte, ma addirittura fulminate nel nulla... »(G. Tomasi di Lampedusa, $l l$ Gattopardo, Milan, 1958, p. 96).

* Se da queste attitudini di Angelica si deducesse che essa amava Tancredi, ci si sbaglierebbe... però, pur non amandolo, essa era, allora, innamorata di lui, il che è assai differente... " (G. Tomasi di Lampedusa, Il Gattopardo, p. 97).

"...but don't take me as a general example, probably I'm just a special case : one of the men who like women, but don't love women... " (D.H. Lawrence, Lady Chatterley's Lover, Londres, 1960, p. 58).

\section{Contextes non synonymiques}

"...neque enim conferendum esse gallicum cum Germanorum agro, neque hanc consuetudinem uictus cum illa comparandam... (Caesar, De bello gallico 1,31 ).

"Ego sum lux mundi : qui sequitur me non ambulat in tenebris, sed habebit lumen vitae. $\gg(\mathrm{Jn} 8,12)$.

"Maria autem stabat ad monumentum foris, plorans : Dum ergom fleret, inclinavit se, et prospexit in monumentum... $\gg(\mathrm{Jn} 20,11)$.

« Parlons plutôt de ma courtoisie. Elle était célèbre et pourtant indiscutable. La politesse me donnait en effet de grandes joies. (A. Camus, la Chute, Paris, 1956, p. 28).

"Ce n'était pas la Vérité, c'était sa mort qui lui parlait par ma bouche. Rien d'étonnant si le fade bonheur de mes premières années a eu parfois un goût funèbre : je devais ma liberté à un trépas opportun, mon importance à un décès très attendu. 》 (J.-P. Sartre, les Mots, p. 20).

* Job lui répondit: « Tu parles comme une folle. Si nous accueillons le bonheur comme un don de Dieu, comment ne pas accepter de même le malheur!» En toute cette infortune, Job ne pécha point en paroles. 》 (Bible de Jérusalem, p. 602).

a Es una enormidad - dice don Francisco - porque con ello quedarían alterados, subvertidos, derruídos, los fundamentos del orden social. - ¿Y porqué iban a quedar destruídos los fundamentos del orden social? " (Azorín, Don Juan, Madrid, 1957, p. 64). 
Da una quarantina d'anni Fontamara non ha un curato. La parrocchia ha unà rendità troppo piccola per mantenere un prete: la chiesa è solita ad aprirsi solo nelle grandi solennità, quando dal capoluogo viene un sacerdote per leggere la messa e spiegarci il Vangelo. " (I. Silone, Fontamara, Milan, 1953, p. 31).

"French was the language of the court and the upper classes, English the speech of the mass of the people. Can we define, however, the position of the two languages more specifically ?» (A.C. Baugh, A History of the English Language, $2^{\circ}$ éd., Londres, 1960, p. 143).

"Wir wissen es selbst, unser Aufbau hat seine schwachen Stellen, aber er hat auch seine starken Seiten. » (S. Freud, Der Mann Moses und die monotheistische Religion, Francfort, 1964, p. 53).

"Die Intoleranz der Massen äussert sich merkwürdigerweise gegen kleinere Unterschiede stärker als gegen fundamentale Differenzen. " (S. Freud, Der Mann Moses..., p. 119).

« Der Unterricht, den wir geniessen, besteht hauptsächlich darin, uns, Geduld und Gehorsam einzuprägen, zwei Eigenschaften, die wenig oder gar keinen Erfolg versprechen. Innere Erfolge, ja. Doch was hat man von solchen? Geben einem innere Errungenschaften zu essen? » (R. Walser, Jakob von Gunten, p. 5).

"Willigis, der unterdessen aufgestanden und auch seiner Schwester behilflich gewesen war, sich zu erheben, sagte darauf... (Th. Mann, Der Erwählte, p. 54).

"An mehreren Tischen wurden Photographien herungezeigt, neue, selbst angefertigte Aufnahmen ohne Zweifel... " (Th. Mann, Der Zauberberg, Francfort, 1956, p. 42).

"Keine einzige sprache ist bis zum punkt der vollkommenheit gelant; eine ideale sprache würde immer dieselbe sache durch dasselbe, ähnliche dinge durch ähnliche ausdrucksmittel bezeichnen... »(O. Jespersen, dans la traduction allemande de son livre Language, p. 433).

Ce passage est particulièrement intéressant parce que l'auteur, au moment même de postuler qu'une chose identique doit toujours être désignée par un mot identique, emploie deux mots différents; dans l'original anglais nous trouvons en effet deux fois le même mot :

"...an ideal language would always express the same thing by the same, and similar things by similar means..." (Language. Its nature, Development and Origin, Londres, 1954, p. 442).

Il y a aussi des contextes non synonymiques dans lesquels la différence des mots est justement mise en relief :

" Jack : I wanted to be engaged to Gwendolen, that is all. I love her.

Algernon : Well, I simply wanted to be engaged to Cecily. I adore her.

Jack: There is certainly no chance of your marrying Miss Cardew.

Algernon : I don't think there is much likelihood, Jack, of you and Miss Fairfax being united. " (O. Wilde, The Importance of Being Earnest, Act II ; dans ce passage la variation synonymique se trouve même dans les noms propres).

"Therefore, you clown, abandon, - which is in the vulgar, leave - the society which in the boorish is company, - of this female, - which in the common is woman; which together is, abandon the society of this female, or, clown, thou perishest; or, to thy better understanding, diest; or, to wit, I kill thee, make thee away, translate thy life into death. * (Shakespeare, As You Like It, V, I; ce passage a déjà été cité par Jespersen, Growth and Structure of the English Language, Oxford, 1960, p. 91, et Ullmann, Principles, p. 109).

Jusqu'ici, nous n'avons parlé que des synonymes dans la parole. En ce qui concerne les synonymes comme éléments de la langue, c'est précisément ceux-ci que la «synonymique » pense prendre pour objet. En réalité la situation n'est pas si simple. La «synonymique »crée, par son procédé même, sans le vouloir, un 
contexte «synonymique ». Elle cherche les différences entre les synonymes. $\mathrm{Vu}$ qu'elle les aborde avec cette intention, elle a déjà posé l'implicat «A diffère de B ». Comme elle a toujours posé cet implicat, il ne peut y avoir pour elle des mots au contenu absolument identique : elle trouve toujours des distinctions, car elle suppose, dès l'abord, l'existence de différences. Elle examine bien les synonymes, comme appartenant à la langue, c'est-à-dire qu'elle les décrit comme existant indépendamment de leur emploi dans la parole et avant celui-ci, comme simplement « virtuels », mais elle le fait - sans s'en rendre compte - dans une actualisation spécifique, produite par son propre procédé, qui correspond au contexte «synonymique » dans la parole. La synonymique fait surgir ainsi une situation linguistique tout à fait particulière et se laisse guider par elle en développant ses subtiles distinctions.

Quelles conclusions peut-on tirer de ce que nous avons dit? Nous relevons quatre points, qui nous paraissent importants.

Primo : Ce résultat révèle la faiblesse de principe qui pèse au départ sur la synonymique traditionnelle telle qu'elle a été inaugurée par Girard. Cette synonymique est dominée, d'une manière plus ou moins explicite, par une doctrine très vieille, déjà établie par les Anciens, celle du «mot propre» (verbum proprium). La thèse est, depuis l'évolution radicale qu'elle subit après le classicisme français, celle-ci : pour chaque contenu particulier, qui cherche son expression, il n'existe qu'UNE SEULE forme linguistique qui lui revient de droit. La formulation classique de cet idéal, dans le domaine du français, est de La Bruyère : "Entre toutes les différentes expressions qui peuvent rendre une seule de nos pensées, il n'y en a qu'une qui soit la bonne. On ne la rencontre pas toujours en parlant ou en écrivant ; il est vrai néanmoins qu'elle existe, que tout ce qui ne l'est point est faible et ne satisfait point un homme d'esprit qui veut se faire entendre ${ }^{17} . \gg \mathrm{La}$ langue est considérée ici par analogie avec un instrument de musique, qui posséderait pour chaque contenu à exprimer une «touche» particulière. La synonymique serait chargée, précisément, de découvrir cette «touche» une fois pour toutes et de la rendre ainsi disponible. Cette conception considère le mot comme une simple étiquette, collée sur un certain contenu : si ce contenu apparaît dans la parole, le mot correspondant ne peut pas manquer, et vice versa. Une pareille conception ne peut être acceptée. La doctrine du «mot propre» s'adapte à la rigueur à ces contextes singuliers que nous appelons «synonymiques». Ce n'est que par rapport à eux que les prétentions descriptives et normatives avancées par la synonymique ont un sens. Voici donc l'erreur de cette synonymique et ce qui fait qu'elle induit en erreur : séduite par son propre point de départ, elle ne voit plus que des contextes «synonymiques»; par conséquent, elle pense qu'on doit parler toujours et exclusivement selon les conditions de pareils contextes. La critique que Rivarol, dans son compte rendu des «Synonymes françois» de Roubaud, adressait aux

17. Caractères, I, 17. Il est intéressant de comparer avec ce passage l'opinion très différente de Rousseau : '...il n'y a point de langue assez riche pour fournir autant de termes, de tours et de phrases, que nos idées peuvent avoir de modifications... Malgré cela, je suis persuadé qu'on peut être clair, mêrme dans la pauvreté de notre langue, non pas en donnant toujours les mêmes acceptions aux mêmes mots, mais en faisant en sorte, autant de fois qu'on emploie chaque mot, que l'acception qu'on lui donne soit suffisamment déterminée par les idées qui s'y rapportent, et que chaque période où ce mot se trouve lui serve, pour ainsi dire, de définition *. (Euvres complètes, éd. Musset-Pathay, III (1823), p. 160). 
adeptes de la synonymique, va déjà dans ce sens. Ses observations contiennent elles-mêmes une intéressante différenciation de synonymes : "Voilà sur quoi ces Messieurs [les synonymistes] ont toujours glissé, et c'est là pourtant le grand mystère du langage. Un mot par lui-même n'est rien qu'un assemblage de lettres; mais une expression est tout : c'est d'elle que les mots attendent la vie. L'expression est une assemblée plutôt qu'un assemblage de mots : elle les réunit et les allie pour peindre un sentiment, une image, une pensée ${ }^{18}$. $\gg$ Il est évident que Rivarol n'aurait pas été d'accord avec une linguistique qui se base exclusivement sur le principe de la « compositionnalité ».

Secundo : La complète identité de contenu est un phénomène de la parole (performance), elle n'est pas un phénomène de la langue («compétence »). Dans la langue, il n'y a que la similitude qui peut être plus ou moins grande. Notre distinction entre les deux types de contextes révèle immédiatement pourquoi la langue n'a pas besoin de mots aux sens absolument identiques : elle n'en a pas besoin, parce que, comme nous l'avons montré, elle a toujours la possibilité de créer, grâce au contexte "non synonymique », une identité totale de contenu dans ses énoncés.

Tertio: Quand la synonymie est définie, selon notre proposition, comme "similitude des contenus », elle ne peut être conçue comme le contraire de la polysémie. D'habitude, on distingue, comme nous l'avons dit, sur un même niveau, deux types opposés de ce que l'on nomme la «signification multiple »: le type « une forme phonique/plusieurs contenus» (polysémie) et le type «plusieurs formes phoniques/un contenu » (synonymie). Mais vu que ce dernier cas n'apparaît pas dans la langue, mais seulement dans la parole, dans des conditions spécifiques, on ne peut pas considérer les deux phénomènes comme étant sur le même niveau. Si les contenus des synonymes dans la langue ne sont pas identiques, on ne peut parler d'un type particulier «signification multiple » opposé à la polysémie. Alors, il importe peu, en ce qui concerne le rapport d'évocation réciproque entre la forme et le contenu - on peut comprendre ainsi la signification -, si les contenus sont très ressemblants, s'ils ont des points de comparaison ou s'ils sont totalement disparates. Dans ce cas, la synonymité n'est que le rapport entre des contenus semblants. Il n'y a donc — dans la langue - qu'un type de « signification multiple $\gg$ : la polysémie. Ce type n'est d'ailleurs pas totalement absent dans la parole ${ }^{19}$.

Quarto : Si la synonymique, par contre, se contente de l'affirmation toujours répétée, qu'il n'existe « au fond» pas de mots aux contenus identiques, elle non plus n'a pas totalement raison. Comme nous l'avons montré, cette affirmation est correcte, pour autant qu'elle s'applique à la langue dans un sens sémasiologique; elle est fausse quand elle porte sur la parole — dans un sens onomasiologique. Si l'on part de ce qui doit être exprimé et que l'on considère à cet effet les moyens linguistiques disponibles, il faut toujours tenir compte du fait qu'une même chose peut être exprimée d'une façon ou d'une autre, et donc que ce qui a été effectivement dit, aurait pu être dit autrement. La vieille rhétorique, dont Girard et ses successeurs cherchaient précisément à se détacher, le savait bien : on y trouve au

18. « Sur les Synonymes françois de M. l'abbé Roubaud », Euvres complètes, t. II, p. 186.

19. Cf. par contre, S. Ullmann, Principles, p. $106 \mathrm{~s}$. 
centre, au sujet des synonymes, l'idée de la copia verborum et par conséquent le de eadem re varie dicere. La pluralité des moyens d'expression disponibles dans chaque cas est un des faits fondamentaux de nos langues et dépasse, naturellement, la synonymie : il est possible de confier, dans un acte concret d'expression linguistique, le même contenu, la même fonction, à des formes différentes. Comme ce fait essentiel comprend le phénomène de la synonymie, et comme la synonymie n'en est qu'une manifestation particulière, bien que très importante, on devrait introduire ici un terme plus général : le terme de POLYMORPHIE. Je reprends ce terme, qui s'applique à tous les domaines de la langue - donc pas uniquement au côté lexical -, de Mario Wandruszka ${ }^{20}$. La polymorphie, le fait que plusieurs formes soient prêtes à accueillir le même contenu, serait alors - en considérant la parole - le phénomène opposé à la polysémie.

La polymorphie, phénomène essentiel pour tout acte linguistique, a pour conséquence que chaque énoncé concret doit être précédé d'un cHorx entre les formes disponibles : chaque énoncé est le résultat d'une série de décisions électives, qui se réalisent principalement dans le domaine du "préconscient ». Au sujet de ces «décisions » on doit retenir deux choses : d'abord, que la même chose peut être dite avec des moyens différents, donc qu'un choix est possible; ensuite, que ces différents moyens, comme la synonymie nous le montre précisément, ne sont pas équivalents à tous les égards, qu'il n'est donc pas absolument indifférent finalement de choisir un moyen ou un autre. Ces deux faits, la possibilité du choix et le fait que le choix n'est pas indifférent, créent les conditions pour l'existence du «style », en prenant ce terme dans son sens le plus large. Il est indiscutable que l'existence du style présuppose nécessairement qu'on puisse dire DIFFÉREMMENT ce qu'on dit. Car la possibilité de ce «différemment » permet au sujet parlant de s'introduire lui-même dans sa manière de parler, d'y transposer son caractère, réel ou adopté. Chaque personne qui parle s'exprime elle-même dans ce qu'elle dit, qu'elle le veuille ou non, et c'est là que se montre - ou que peut se montrer - le style. C'est exactement ce que veut dire le mot si célèbre de Buffon que Pierre Guiraud commente spirituellement de la sorte : "Quoi qu'en dise Buffon, le style c'est l'homme ${ }^{21}$.»

Ces langues, que la logique traite, avec une expression peu heureuse (mais : in verbis simus faciles), de langues «naturelles», ne remplissent ainsi d'aucune manière une des conditions fondamentales que cette même logique impose à ces langues « artificielles »: le rapport unique et réversible dans la relation entre le signifiant et le signifié. Nous retrouvons à tout moment la polysémie et la polymorphie dans les actes linguistiques de la langue "naturelle », c'est-à-dire de la LANGIUE HISTORIQUE. Comme les langues artificielles n'empêchent pas seulement la polysémie, mais aussi la polymorphie, elles sont nécessairement des langues sans style, c'est-à-dire qu'elles sont - dans un sens précis, point du tout dépréciatif des LANGuES inHUMaINES. En effet, ceux qui composent et utilisent ces langues ont l'intention - une intention légitime dans leur domaine - d'éliminer le sujet parlant de ses actes linguistiques. On veut empêcher de cette manière que quelque chose du sujet ne se glisse subrepticement dans la parole, dépassant et brouillant

20. Cf. M. Wandruszka, Sprachen, vergleichbar und unvergleichlich, Munich, 1969, passim. 21. P. Guiraud, la Stylistique, Paris, 1954, p. 5. 
ainsi le pur contenu de réalité de l'énoncé. Pour les langues historiques cependant, l'ouverture à l'égard du côté subjectif n'est pas seulement caractéristique, mais constitutive : rien d'humain ne leur est étranger. Cette ouverture vers le sujet parlant avec tout son conditionnement historique, social, culturel et psychique n'est pas un défaut. C'est elle précisément qui fait ce que l'on nomme la « richesse » de la langue. Les synonymes sont une des manifestations de cette richesse, certainement la plus importante : ils contiennent les innombrables distinctions subtiles qui ont été gravées dans la terne matière des formes phoniques par l'expérience du monde et par celle d'une société à travers son histoire.

Hans-Martin Gauger 